\title{
Estudio de un absorbedor basado en metamaterial quiral
}

\author{
Study of absorber based on chiral metamaterial
}

\author{
Perla Espinosa Diaz $\quad$ Diego Torres Cabezas ${ }^{2} \quad$ H. Torres-Silva ${ }^{3}$ \\ Recibido 6 de septiembre de 2010, aceptado 18 de marzo de 2011 \\ Received: September 6, $2010 \quad$ Accepted: March 18, 2011
}

\section{RESUMEN}

\begin{abstract}
El presente trabajo estudia el comportamiento electromagnético de un dispositivo absorbedor con estructura metamaterial quiral. El dispositivo propuesto consiste en una estructura de planos paralelos, con rosetas metálicas implantadas, físicamente separadas por capas dieléctricas. Para demostrar el efecto absorbedor de tal estructura, se ejecuta una simulación básica, en la cual se hace incidir un haz gaussiano sobre el metamaterial quiral. Los resultados muestran que, para efectos prácticos, ocurre absorción total. Este comportamiento hace que la estructura propuesta se comporte como un "agujero negro espacial".
\end{abstract}

Palabras clave: Quiral, metamaterial, agujero negro, absorción, refracción negativa.

\begin{abstract}
The electromagnetic behavior of an absorber device with chiral metamaterial structure is studied. The proposed device is a structure formed by parallel planes with implanted metallic rosettes, physically separated by dielectric layers. In order to show the absorbing properties of such structure, a basic simulation is run, in which a gaussian beam is fed incident to the chiral metamaterial. Results show that there is, for practical purposes, full absorption. This behavior makes the proposed structure behave as a "spatial black hole".
\end{abstract}

Keywords: Chiral, metamaterials, black hole space, absorption, negative refraction.

\section{INTRODUCCIÓN}

Los metamateriales están compuestos por materiales artificiales diseñados para obtener, simultáneamente, una permitividad eléctrica $(\varepsilon)$ y permeabilidad magnética $(\mu)$ negativas, ello permite obtener materiales con propiedades electromagnéticas inusuales, incluyendo índice de refracción negativo (lo que se traduce en una inversión de la ley de Snell) y desviación del efecto Doppler y de la radiación Cherenkov [1-3]. Estas características han dado lugar a potenciales aplicaciones en diferentes áreas, tales como: mantos de invisibilidad electromagnética, imágenes de súper resolución, concentradores electromagnéticos, almacenadores de luz, etc. Un medio metamaterial presenta un comportamiento opuesto a las de un medio convencional, ya que el vector de campo eléctrico $\vec{E}$, el vector de campo magnético $\vec{H}$ y el número de onda $\vec{k}$ forman una tríada zurda, lo cual supone que la luz se propague en sentido contrario al flujo de energía, representado por el vector de Poynting $\vec{S}$. Esta situación se muestra en la Figura 1; en un medio metamaterial el vector de Poynting y el número de onda tienen sentidos opuestos, mientras que en un medio convencional tienen el mismo sentido.

1 Escuela Universitaria de Ingeniería Eléctrica-Electrónica. Universidad de Tarapacá. Casilla 6-D. Arica, Chile. E-mail: perlae@gmail.com

2 Departamento Tecnologías de Información. Dirección del Trabajo, Agustinas 1253, Of. 509. Santiago, Chile. E-mail: torresdc@hotmail.com

3 Instituto de Alta Investigación. Universidad de Tarapacá. Casilla 6-D. Arica, Chile. E-mail: htorres@uta.cl 


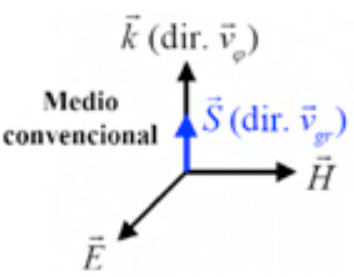

(a)

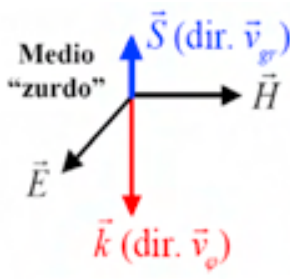

(b)
Figura 1. Características de (a) un medio convencional y (b) un medio metamaterial.

Consecuentemente, en ambos medios la energía se propaga alejándose de la fuente de radiación, pero en metamateriales los frentes de ondas se desplazan hacia la fuente. Por eso en dichos medios las ondas se denominan ondas de retroceso, donde la velocidad de fase $\vec{v}_{p}$ y la velocidad de grupo $\vec{v}_{g}$ tienen sentidos opuestos. Este efecto es compensatorio en la estructura que se propone.

Por otro lado, los medios quirales poseen la habilidad de rotar el plano de polarización de la luz (girotropía) [4-5]. Este efecto en la actualidad es ampliamente usado en distintas áreas de las ciencias. El desarrollo de metamateriales, con índice de refracción negativa, ha impulsado el interés de medios girotrópicos, ya que las respuestas eléctricas y magnéticas de estas estructuras son necesarias para obtener la refracción inversa. Sin embargo, no se han identificado materiales girotrópicos naturales con índice de refracción negativa, es por ello que se ha optado por la construcción de medios girotrópicos artificiales.

En este trabajo se estudiará el comportamiento electromagnético de una estructura metamaterial quiral. La estructura propuesta está compuesta por patrones metálicos insertos en forma períodica en el medio dieléctrico como se aprecia en la Figura 2.

La forma elegida para el patrón metálico es la hélice o también conocida como roseta [5], mostrada en la Figura 3.

Este dispositivo actuará como un absorbedor, en la frecuencia de microondas, atrapando las ondas electromagnéticas que inciden en él, similar al comportamiento de un agujero negro en el rango de las microondas. Este fenómeno ha sido ampliamente estudiado en el rango óptico [6]. Extrapolando el

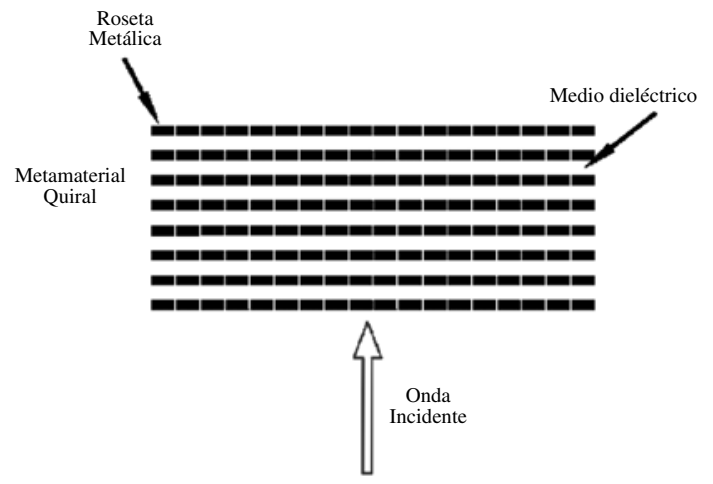

Figura 2. Diagrama del medio metamaterial conformado por múltiples patrones metálicos planos.
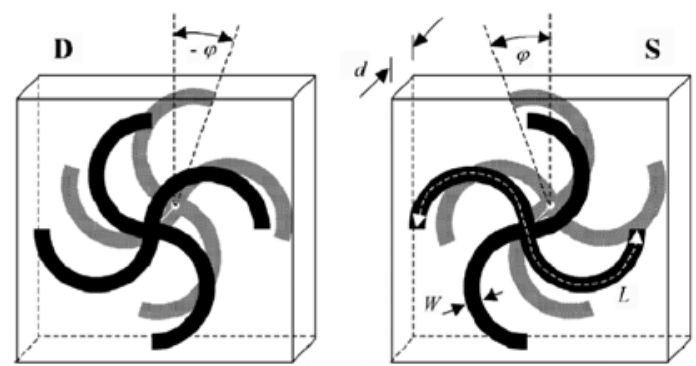

Figura 3. Estructura de capas compuesta por una hélice plana metálica separada por una capa dieléctrico de ancho d para ondas helicoidales a la derecha y a la izquierda respectivamente.

funcionamiento de este fenómeno a todo el espectro, permite disponer de un modelo de estudio que amplíe el conocimiento de las características de los agujeros negros espaciales, donde la luz en forma de vórtices (ondas de extrema quiralidad) es atrapada.

En las secciones siguientes se presenta el modelo matemático que modela el comportamiento físico de la estructura metamaterial quiral y las simulaciones realizadas para demostrar el efecto de absorción casi total que tal dispositivo presenta en la región de microondas.

\section{TEORÍA DE METAMATERIALES QUIRALES}

El medio metamaterial quiral, compuesto de capas paralelas con arreglos de rosetas metálicas, puede ser modelado por el formalismo de Born-Fedorov [7]. 


$$
\begin{aligned}
\vec{D} & =\varepsilon(\vec{E}+T \nabla \times \vec{E}) \\
\vec{B} & =\mu(\vec{H}+T \nabla \times \vec{H})
\end{aligned}
$$

donde $T$ es el factor quiral, $\mu$ es la permeabilidad magnética y $\varepsilon$ es la permitividad eléctrica normalizada en el medio isótropo. Efectuando una transformación donde las ecuaciones de Maxwell en el vacío están dadas por: $\nabla \times \vec{E}=-\mu \frac{\partial \vec{H}}{\partial t}$ y $\nabla \times \vec{H}=\varepsilon \frac{\partial \vec{E}}{\partial t}$ y considerando que todos los campos tienen una dependencia del tiempo $e^{j \omega t}$ se obtiene una forma más conveniente para las ecuaciones (1), conocidas como el formalismo de Tellegen [4]:

$$
\left(\begin{array}{c}
\vec{D} \\
\vec{B}
\end{array}\right)=\left(\begin{array}{cc}
\varepsilon_{0} \varepsilon_{r} & -j T k / c \\
j T k / c & \mu_{0} \mu_{r}
\end{array}\right)\left(\begin{array}{c}
\vec{E} \\
\vec{H}
\end{array}\right)
$$

donde $k$ es el número de onda, $c$ es la velocidad de la luz, $\varepsilon_{0}$ es la permitividad del vacío, $\mu_{0}$ permeabilidad del vacío, $\varepsilon_{r}$ es la permitividad relativa y $\mu_{r}$ es la permeabilidad relativa. Con la condición de que $\varepsilon_{0} \varepsilon_{r}=\mu_{0} \mu_{r}$, las autosoluciones son ondas circularmente polarizadas a la derecha (+) y a la izquierda (-), respectivamente, donde el número de onda es dado por:

$$
k_{ \pm}=\omega(\sqrt{\mu \varepsilon} \pm \kappa)
$$

En términos del formalismo de Born-Fedorov es:

$$
k_{ \pm}=k_{0} /\left(1 \pm k_{0} T\right)
$$

$\operatorname{con} \kappa=T k / c$ y $k_{0}=\omega / c$.

La igualdad entre las ecuaciones (3) y (4) se encuentra si se escribe el campo eléctrico en términos del potencial eléctrico $\phi$ y magnético $\vec{A}$, de manera que $\vec{E}=-j \omega \vec{A}-\nabla \phi$ y el campo magnético es dado por $\vec{B}=\nabla \times \vec{A}$. Substituyendo estas expresiones en las ecuaciones de Maxwell se tiene, en el calibre de Lorentz, una ecuación para la divergencia

$$
\nabla \cdot \vec{A}=-j \frac{\omega \mu \varepsilon}{1-k_{o}^{2} T^{2}} \phi
$$

y una ecuación de onda para $\vec{A}$ $\nabla^{2} \vec{A}+\frac{k_{0}^{2}}{1-k_{0}^{2} T^{2}} \vec{A}+2 \frac{\omega^{2} \mu \varepsilon T}{1-k_{o}^{2} T^{2}}(\nabla \times \vec{A})=0$

Se sabe que $k=\sqrt{k_{x}^{2}+k_{y}^{2}+k_{z}^{2}}$. En coordenadas rectangulares se tiene $k_{x}=k \sin \theta, k_{y}=0 \quad \mathrm{y}$ $k_{z}=k \cos \theta$, por lo que la ecuación de onda descrita (6) puede ser descompuesta en la siguiente matriz:

$$
\left(\begin{array}{ccc}
-k^{2}\left(1-k_{0}^{2} T^{2}\right)+k_{0}^{2} & -2 j k_{0}^{2} k T \cos \theta & 0 \\
2 j k_{0}^{2} k T \cos \theta & -k^{2}\left(1-k_{0}^{2} T^{2}\right)+k_{0}^{2} & -2 j k_{0}^{2} k T \sin \theta \\
0 & 2 j k_{0}^{2} k T \sin \theta & -k^{2}\left(1-k_{0}^{2} T^{2}\right)+k_{0}^{2}
\end{array}\right)\left(\begin{array}{c}
A_{x} \\
A_{y} \\
A_{z}
\end{array}\right)=0
$$

La solución del determinante permite obtener la relación de dispersión para el campo longitudinal,

$$
k^{2}\left(1-k_{0}^{2} T^{2}\right)=k_{0}^{2}=\omega^{2} / c^{2} \Rightarrow k= \pm k_{0} / \sqrt{1-k_{0}^{2} T^{2}}
$$

que tiene directa relación con la formación de partículas creadas por la estructura tipo vórtices de los campos cuando la quiralidad $\mathrm{T}$ es intensa. Para los propósitos de este trabajo, esta relación de dispersión de ondas longitudinales no será discutida, indicando apenas que es importante en los modos de luz que se generan en el interior de agujeros negros.

Por otro lado, la relación de dispersión para el campo transversal es:

$$
\left(-k^{2}\left(1-k_{0}^{2} T^{2}\right)+k_{0}^{2}\right)^{2}-4 k_{0}^{4} k^{2} T^{2}\left(\sin ^{2} \theta+\cos ^{2} \theta\right)=0
$$

$\Rightarrow k=k_{ \pm}= \pm k_{0} /\left(1 \pm k_{0} T\right)$ que es equivalente a las expresiones (3) y (4).

Volviendo a las Figuras 2 y 3, se considera que las estructuras tienen pérdidas por las inserciones metálicas tipo roseta. Además se supone que el índice de refracción y el módulo de la impedancia para estos medios, tratados como medios complejos, están dados por:

$$
n_{ \pm}=-\frac{j}{k_{0} d} \ln \left(\frac{1}{\tau_{ \pm}}\left(1-\frac{Z-1}{Z+1} r\right)\right)
$$




$$
Z=\sqrt{\frac{(1+r)^{2}-\tau_{+} \tau_{r-}}{(1-r)^{2}-\tau_{+} \tau_{-}}}
$$

Aquí, $n_{ \pm}$es proporcional a $k_{ \pm}=k_{0} /\left(1 \pm k_{0} T\right), r$ y $\tau$ son los coeficientes de reflexión y transmisión, respectivamente, $k_{0}$ es el vector de onda en el vacío y $d$ es el espesor de la estructura tipo roseta. La raíz y la función logaritmo deben ser cuidadosamente escogidas de acuerdo al principio de conservación de energía, $\operatorname{Re}(Z)>0$. El valor negativo de $n_{ \pm}$, que determina el carácter de metamaterial, es producto de la quiralidad si $k_{0} T$ es negativo y mayor que uno para $k_{+}, \mathrm{o} k_{0} T$ es positivo y mayor que uno para $k_{-}$. Dentro de la estructura se tienen distribuciones de ondas con $k_{+} \mathrm{y} k_{-}$.

\section{SIMULACIONES}

A fin de ilustrar y comprobar los fenómenos teóricos previstos, es decir tener una alta absorción de las ondas incidentes, se presenta una simulación computacional en base al modelo teórico dado por las ecuaciones (7) y (8). Se excita la estructura metamaterial quiral con un haz de ondas que simula una gaussiana, con frecuencia central de alrededor de $12 \mathrm{GHz}$. La incidencia de la onda está correlacionada con el coseno del ángulo. Las tasas de absorción, transmisión y reflexión están normalizadas por la potencia de entrada $E_{i n c}^{2} / 2 \eta_{0}=1$, de modo que dichas tasas estén acotadas en [0,1]. La expresión $\varepsilon_{0} \varepsilon_{r}=\mu_{0} \mu_{r}$ es vital para la adaptación de impedancia. Se permite una pequeña pérdida en la estructura para que la onda se vaya atenuando en el dispositivo fruto de las múltiples reflexiones internas, o sea, $\operatorname{Im} \varepsilon_{r} \ll \operatorname{Re} \varepsilon_{r}, \operatorname{Im} \mu_{r} \ll \operatorname{Re} \mu_{r}$. Para los efectos de ilustración, el valor absoluto de $k_{0} T$ es del orden de $1,1\left(\left|k_{0} T\right| \sim 1,1\right)$. La situación de estado estacionario ocurre luego de algunos ciclos de la onda incidente. La respuesta del dispositivo se evalúa mediante el valor de los coeficientes de absorción y transmisión. Los resultados obtenidos se muestran en las Figuras 4 y 5 . La Figura 4 presenta el comportamiento del coeficiente de absorción en función de la frecuencia y se observa que el dispositivo absorbe el haz gaussiano casi por completo, con una tasa de aproximadamente $95 \%$.

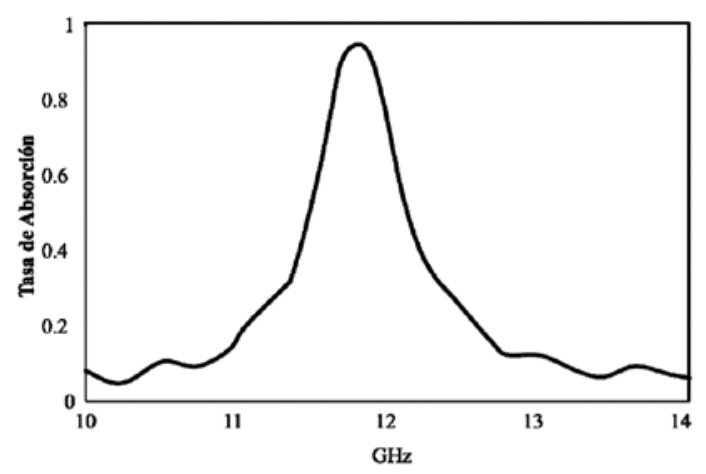

Figura 4. Diagrama de absorción del dispositivo.

La Figura 5 presenta el desempeño de los coeficientes de transmisión y reflexión en función de la frecuencia, y de acuerdo a lo esperado se observa que a la frecuencia de excitación la reflexión, a la entrada del dispositivo, es prácticamente nula, mientras que a su salida, la transmisión es muy pequeña, tendiendo a cero, por lo que se deduce que toda la potencia que ingresó en el dispositivo metamaterial quiral fue absorbida.

Nuestras simulaciones numéricas muestran que la absorción es casi independiente del ángulo de incidencia.

Medidas experimentales de otros autores [8,9], confirman esto último.

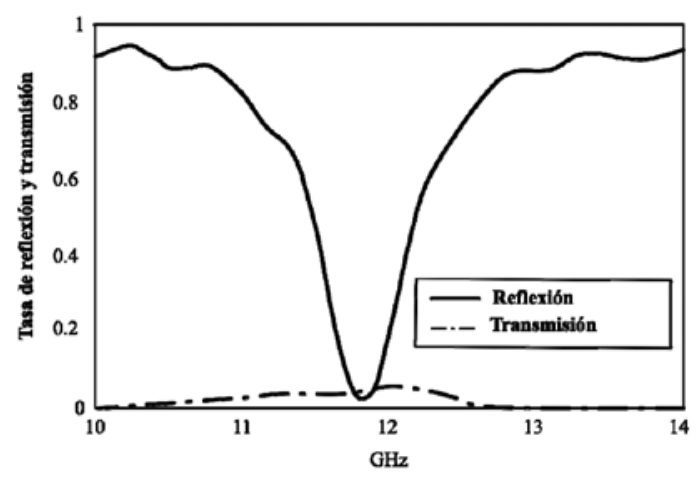

Figura 5. Diagrama de reflexión y transmisión del dispositivo. 


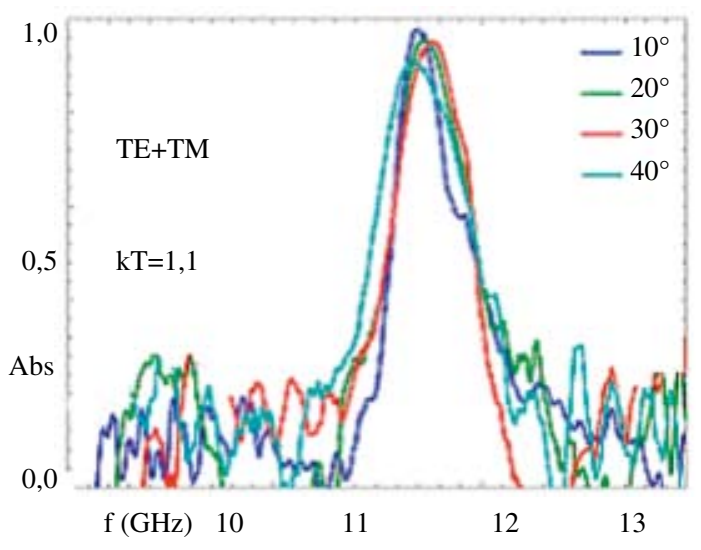

Figura 6. Diagrama de absorción co+n polarización TE y TM simultáneamente para diferentes ángulos de incidencia.

Nuestras simulaciones numéricas (Figura 6), para onda viajera incidente muestran que la absorción es casi independiente del ángulo de incidencia. Las curvas están normalizadas en comparación con aquella que tiene un ángulo de incidencia igual a cero grado $\left(0^{\circ}\right)$. Se consideró una onda mixta de polarización TE+TM porque representa adecuadamente un campo del tipo quiral, $\nabla \times\left(\vec{E}^{T E} \pm i \vec{E}^{T M}\right)=\mp k\left(\vec{E}^{T E} \pm i \vec{E}^{T M}\right)[10]$.

En la zona de máxima absorción, las curvas son similares. Esto reafirma el hecho de que las pérdidas internas del dispositivo son importantes pero no su valor absoluto. Las múltiples reflexiones internas posibilitan la absorción paulatina entre ida y vuelta de la onda atrapada. Posiblemente las oscilaciones de la parte baja de la absorción de las diferentes curvas se deben a que para el cálculo numérico se optó por alrededor de 10 idas y venidas de la onda incidente.

\section{CONCLUSIONES}

De este trabajo se puede concluir lo siguiente:

- La estructura propuesta se comporta como un absorbedor en la región de microonda, ya que al incidir un haz que simula una gaussiana a la frecuencia $12 \mathrm{GHz}$ atrapa casi en su totalidad la señal incidente con una reflexión prácticamente nula. La tasa de absorción del dispositivo metamaterial quiral es de aproximadamente del $95 \%$.

- Debido a la alta tasa de absorción, este dispositivo se comporta como un agujero negro electromagnético atrapando y absorbiendo las ondas circulantes. La revelancia del trabajo es que ampliando el espectro de trabajo del absorbedor se podría conocer más acabadamente el real fenómeno de los agujeros negros espaciales.

\section{REFERENCIAS}

[1] V.G. Veselago. "The Electrodynamics of substances with simultaneously negative values of $\varepsilon$ and $\mu$ ". Soviet Physics Uspekhi. Vol. 10, Issue 4, pp. 509-514. 1968.

[2] J.B. Pendry, D. Schurig and D.R. Smith. "Controlling electromagnetic fields". Science. Vol. 312, Issue 5781, pp. 17801782. 2006.

[3] A. García Aguilar. "Análisis, diseño y prototipo de una lente plana basada en estructuras metamateriales para antenas". Universidad Politécnica de Madrid. Madrid, España. 2008.

[4] S.J. Orfanidis. "Electromagnetic Waves and Antennas". 2010. Fecha de consulta: 17 de mayo de 2010. URL: www.ece.rutgers. edu/ orfanidi/ewa/

[5] A.V. Rogacheva, V.A. Fedotov, A.S. Schwanecke and N.I. Zheludev. "Giant Gyrotropic due to Electromagnetic-Field Coumpling in a Bilayered Chiral Structure". Physical Review Letters. Vol. 97, Issue 17, 177-401. October, 2006.

[6] E.E. Narimanov and A.V. Kildishev. "Optical black hole: Broadband omnidirectional light absorber". Applied Physics Letters. Vol. 95, Issue 4, 041106. 2009.

[7] H. Torres Silva. "Chiral waves in a metamaterial medium". Ingeniare. Revista chilena de ingeniería. Vol. 16, número especial, pp. 119122. 2008. DOI: $10.4067 /$ S0718-33052008 000400017.

[8] B. Zhu, Z. Wang, C. Huang, Y. Feng, J. Zhao and T. Jiang. "Polarization insensitive metamaterial absorber with wide incident angle". Progress In Electromagnetic Research, PIERS. Vol. 101, pp. 231-239. 2010.

[9] Z. Li, H. Caglavan, E. Colak, J. Zhou, C.M. Soukoulis and E. Ozbay. "Coupling effect between two adjacent chiral structure layers" Optics Express. Vol. 18, Issue 6, pp. 53755383. 2010.

[10] W.T.M. Irvine and D. Bouwmeester. "Linked and knotted beams of light" Nature Physics. Vol. 4, pp. 716-720. 2008. 